\title{
VALORACIÓN SOCIOECONÓMICA DE LA EXTRACCIÓN DE GAS MEDIANTE FRACTURACIÓN HIDRÁULICA EN LA REGIÓN DE MURCIA
}

\author{
José M. Martínez-Paz, Francisco Pellicer-Martínez, José Ramón Fernández y Luca \\ Lamonaca \\ Instituto Universitario del Agua y el Medioambiente \\ Universidad de Murcia.
}

\begin{abstract}
RESUMEN
La extracción de gas no convencional mediante fracturación hidráulica (fracking) es una técnica controvertida, ya que a sus beneficios sociales y económicos se contraponen sus potenciales riesgos ambientales y sobre la salud humana. En el norte de la Región de Murcia, al igual que en otras zonas de España, se han concedido recientemente permisos de investigación y prospección para la posible explotación de yacimientos de gas mediante fracking, lo que ha originado la aparición de movimientos ciudadanos que abogan por su prohibición. El objetivo central de este trabajo es estudiar el conocimiento, la percepción y la aceptabilidad que tienen los ciudadanos de la Región de Murcia sobre la explotación de esta fuente de energía. Con este fin se realiza una encuesta en la que se implementa el método de la Valoración Contingente. Los resultados informan, entre otros, que algo más de dos tercios de la población estarían a favor de su implementación, condicionado a que salud y el medioambiente sean salvaguardados. A su vez cada familia murciana se muestra dispuesta a contribuir, en media, con $15 € /$ año como sobrecoste en el recibo eléctrico para fijar una moratoria en la explotación.
\end{abstract}

Palabras clave: fracking; gas esquisto; valoración contingente; Murcia.

\section{SOCIO-ECONOMIC ASSESSMENT OF GAS EXTRACTION BY FRACKING IN THE REGION OF MURCIA.}

\begin{abstract}
Extraction of unconventional gas through hydraulic fracturing, the so-called "fracking", is a highly controversial debate, considering on the one hand economic benefits and on the other hand its environmental impacts, and risks for public health. Recently, relevant Policy makers approved applications to allow the exploration of gas resources that could be extracted through fracking in the North Murcia region, likewise in other parts of Spain. Such approvals caused the formation of local groups that oppose fracking. The main objective of this investigation is to explore the knowledge, public perception and social acceptability of the citizens of the Murcia Region upon this technique for energy generation. A survey has been developed to address such research objective, undertaking also a contingent valuation on the prohibition of implementation fracking in the region. Among others, the findings show that more than $2 / 3$ of the survey respondents are in favour of the implementation of such technique provided that the environment and public health would be effectively safeguarded. Furthermore, it is estimated that each household, on average, would be willing to pay approximately $€ 15$ per year for their
\end{abstract}

Fecha de recepción: 27 de julio de 2014. Fecha de aceptación: 19 de agosto de 2015. 
energy bill in order to prohibit or postpone the exploitation of unconventional gas through fracking in the region.

Key words: fracking; shale gas; contingent valuation; Murcia.

\section{INTRODUCCIÓN}

La extracción de gas no convencional mediante la técnica de la fracturación hidráulica, conocida con el término "fracking", se basa en inyectar a gran presión un fluido en el subsuelo para liberar el gas que está atrapado en pizarras y esquistos bituminosos de baja permeabilidad (BOUDET et al., 2014). Para ello, previamente se realiza una perforación vertical que puede alcanzar entre los 2.000 y 3.000 metros de profundidad (GARCÍA-PORTERO, 2012) seguida de una perforación horizontal que continua hasta dar con la ubicación de este tipo de rocas que contienen el gas, denominado gas esquisto o gas no convencional.

La técnica de la fracturación hidráulica ya se usó en 1946 para la explotación del yacimiento petrolífero Hugoton, situado al suroeste del estado de Kansas en los Estados Unidos (MATESANZ, 2013). Es a mitad de los años 90 cuando comienza su expansión y explotación a gran escala (BOUDET et al., 2014) concentrándose en Estados Unidos y Canadá la mayor parte de ellos (GARCÍA-PORTERO, 2012). De hecho, la producción de gas esquisto estadounidense supone casi la cuarta parte de su producción de gas natural (CÁMARA y PENDAS, 2013), lo que ha permitido a este país reducir notablemente su dependencia energética exterior hasta el punto que se espera que en un futuro se convierta en exportador de gas natural (RATNER et al., 2015). México y Argentina son otros dos países donde se prevé un fuerte desarrollo de esta técnica, estimándose que este último puede llegar a ser uno de los mayores productores de gas no convencional tras el descubrimiento en su territorio del yacimiento de "Vaca Muerta", de más de $30.000 \mathrm{Km}^{2}$ y que es uno de los mayores situados fuera de Norte América (EIA, 2013)

La explotación del gas esquisto es un tema de gran actualidad, debido a la controversia que genera su implementación. De un lado reduce la dependencia energética exterior, factor de indudable importancia dada la volatilidad de los precios de petróleo, que junto a la disminución del coste de la energía y a la creación de empleo vienen siendo sus principales ventajas (ROUSU et al., 2015). De otro, sus inconvenientes están asociados a la naturaleza de la técnica empleada y a sus potenciales impactos ambientales, en opinión de muchos expertos no suficientemente evaluados: riesgos sísmicos, cantidad de agua utilizada, contaminación de acuíferos, emisiones de $\mathrm{CO}_{2}$, emisiones de contaminantes, riesgo de escapes de gas (COOLEY y DONNELLY, 2012; RAHM y RIHA, 2012). De todos ellos, el impacto sobre la actividad sísmica resulta uno de los aspectos más controvertidos, por su desconocimiento, y de especial importancia en zonas donde existen fallas sismogénicas activas (WESTAWAY y YOUNGER, 2014). También, preocupa en gran medida los posibles impactos de las sustancias químicas presentes en el fluido inyectado, tanto por su impacto en la salud humana (KASSOTIS et al., 2014) como por su afección al estado de los acuíferos (VENGOSH et al., 2014), en el aire (COLBORN et al., 2014) y/o en el agua de boca (EPA, 2015).

Tras una serie de informes y alegaciones, la Comisión Europea ha renunciado a legislar sobre este tema, y a principios de 2014 presentó una recomendación no vinculante de principios generales para planear los desarrollos de extracción de gas esquisto y sus impactos ambientales, que en la práctica deja vía libre a sus Estados miembros para decidir si quieren explotar o no este gas no convencional (DOUE, 2014). Así, por ejemplo, en la actualidad, mientras Francia y Bulgaria la prohíben, Reino Unido y Polonia son sus más fervientes defensores y planean un fuerte desarrollo de los mismos en los próximos años (SÁNDEZ, 2014).

En España, y dada su elevada dependencia energética exterior, hay un interés creciente por esta fuente energética endógena, habiéndose realizado muy recientemente una primera evaluación de su potencial que indica que los recursos de gas esquisto existentes podrían proveer de gas al país durante 39 años (CÁMARA y PENDAS, 2013). Aunque no existe ninguna explotación activa en España, son más de 100 las zonas con permisos de investigación 
vigentes, los cuales evaluaran la viabilidad técnica y económica de iniciar la extracción ${ }^{2}$. Estos permisos se sitúan en las cuencas Vasco-Cantábrica, Pirenaica, Ebro, Guadalquivir y Bética, siendo la primera de éstas la que dispone de mayor cantidad de gas explotable (ACIEP, 2012). Paralelamente a la concesión de estos permisos ha surgido en España al igual que en todos los estados donde se ha iniciado su implementación, un importante movimiento social de oposición a la explotación de gas esquisto, articulado en ONGs, asociaciones ciudadanas o partidos políticos. Los argumentos generales de esta oposición se basan en que los elevados riesgos ambientales y sanitarios no están claramente definidos, en la falta de estudios rigurosos sobre las reservas potenciales existentes y en la posible insostenibilidad financiera de su explotación a corto plazo, lo que se ha venido a llamar la "burbuja del fracking" (GERLACH, 2013; PEINADO, 2014).

Muestra de la división de opiniones y controversia social que genera la fracturación hidráulica en España es el hecho de que mientras algunas comunidades autónomas han legislado contra la implantación de esta técnica en su territorio (Cantabria, La Rioja, Navarra y Cataluña) y otras han apostado por moratorias temporales (como Andalucía o País Vasco), el gobierno central acaba de aprobar la ley sobre la exploración, investigación y explotación de hidrocarburos (BOE, 2015). A través de esta ley, se han creado incentivos económicos para las comunidades autónomas, municipios y propietarios particulares que desarrollen esta actividad. No obstante, la actual norma nacional sobre Evaluación de Impacto Ambiental (BOE, 2013) impone la obligación de someter a evaluación de impacto ambiental aquellos proyectos relacionados con la exploración, investigación o explotación de hidrocarburos mediante fracturación hidráulica.

En la Región de Murcia, ámbito geográfico del presente trabajo, son cuatro las áreas que tienen permiso de investigación (figura 1). Tres de ellas, denominadas Aries 1, Aries 2 y Leo, son de ámbito nacional al comprender áreas de más de una comunidad autónoma, siendo la cuarta, Escorpio, de ámbito regional. Existe una quinta zona, con un permiso de investigación regional solicitado, denominada Acuario.

La Región de Murcia puede considerarse una zona especialmente sensible a la implantación de esta técnica por dos motivos fundamentales. De un lado, el consumo de agua durante el funcionamiento de la técnica y las posibles afecciones a los recursos hídricos subterráneos, impactos que toman especial importancia en una cuenca semiárida y con acuíferos sobreexplotados como es la del Segura (CHS, 2013) y donde daba la ubicación de los sondeos se podrían ver afectadas masas de agua subterráneas como el sinclinal de Calasparra y los acuíferos de Caravaca, Jumilla-Yecla o Cingla entre otros. De otro, porque el riesgo de inducir movimientos sísmicos, que se achaca a la fracturación hidráulica, se ve agravado en una zona altamente sísmica como es la Región de Murcia, donde el terremoto de Lorca de 2011 es buena prueba de ello (MARTÍNEZ, 2011).

\footnotetext{
${ }^{2}$ Los mapas oficiales de permisos y concesiones de hidrocarburos del Ministerio Industria, Energía y Turismo durante los últimos 10 años pueden consultarse en la referencia MINETUR (2015).
} 
FIGURA 1

Mapa de posición de permisos de investigación y concesiones de explotación en la Región de Murcia, Diciembre 2014.

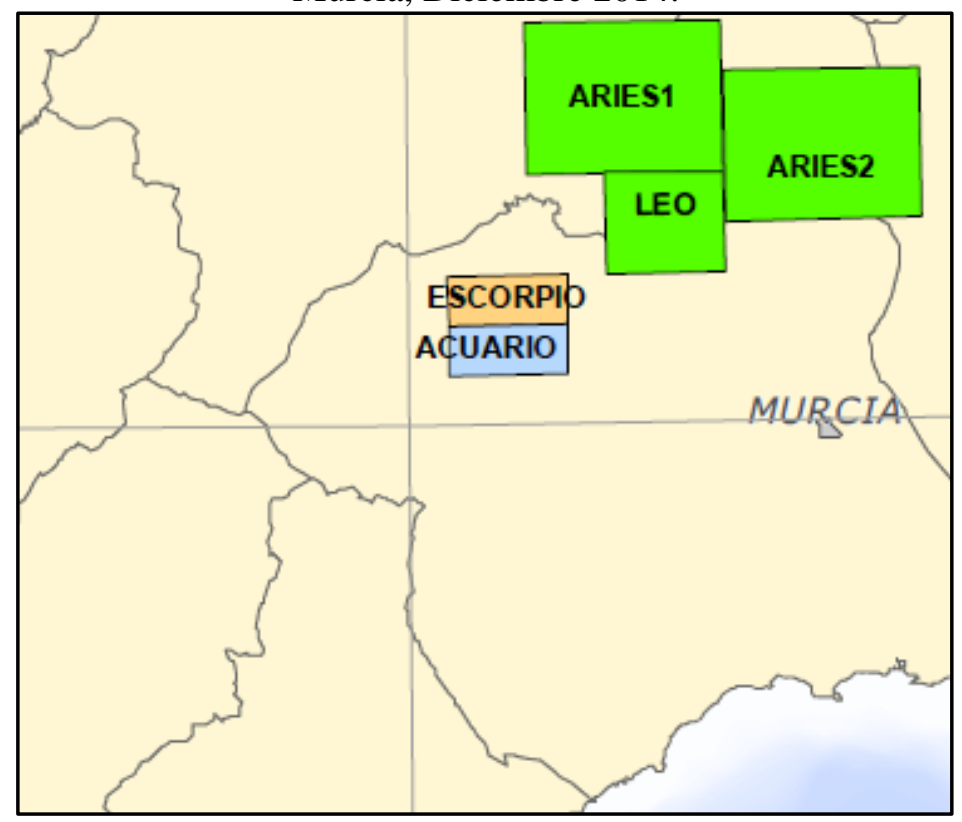

Fuente: MINETUR (2015)

Esta situación de controversia sobre los riesgos y potencialidades de esta fuente de energía hace que en el diseño de sus planes de implementación o de prohibición, sea fundamental conocer la percepción social sobre la misma y cuál es el nivel de aceptación de la población potencialmente afectada (ROUSU et al., 2015). Una revisión de los antecedentes existentes en la literatura científica sobre trabajos que aborden estas cuestiones pone de manifiesto su escasez, y se observa que la mayoría de los estudios se concretan en poblaciones de los Estados Unidos (POPKIN et al., 2013, BOUDET et al., 2014; BENNET y LOOMIS, 2015), no teniendo constancia de ninguno en Europa.

Las instituciones de la Unión Europea realizaron un estudio de consulta pública sobre la percepción de este tipo de energía (EC, 2013), previamente a la promulgación de las recomendaciones sobre su implantación antes mencionadas (DOUE, 2014). En esta consulta, realizada por internet, y que obtuvo más de 22.000 respuestas, se constata que la opinión sobre el desarrollo de la técnica esta equilibrada entre sus detractores y sus defensores, aunque con una gran variabilidad por países.

El presente trabajo tiene como objetivo evaluar el grado de conocimiento y la percepción que tienen los habitantes de la Región de Murcia sobre la extracción de gas no convencional mediante fracturación hidráulica, analizando también la disposición a contribuir económicamente a una moratoria a su implantación, que viene a ser equivalente a dar una valoración monetaria de los riesgos percibidos. Para ello se ha diseñado una encuesta a hogares que incluye, entre otros, un ejercicio de Valoración Contingente (VC).

A esta introducción y enunciado de los objetivos del trabajo le sigue el epígrafe donde se presenta el marco metodológico utilizado y las particularidades para el caso de estudio. El tercer epígrafe recoge los principales resultados obtenidos, terminando el trabajo con una exposición de las conclusiones y recomendaciones que se derivan de los análisis previos.

\section{MATERIAL Y MÉTODOS}

Como ya se ha apuntado en el epígrafe anterior, la metodología aplicada en este estudio es el análisis de una encuesta sobre la valoración y percepción de la fracturación hidráulica a ciudadanos de la Región de Murcia. En dicha encuesta, además de preguntar sobre la técnica, se 
plantearon cuestiones sobre su concienciación ambiental y sobre sus actitudes, percepciones y emociones ante el fenómeno del cambio climático, constando la misma de 20 preguntas estructuradas en 4 bloques temáticos:

- I: Opinión sobre temas sociales, económicos y ambientales generales.

- II: Conocimiento y percepciones del riesgo derivado de la extracción de gas mediante fractura hidráulica

- III: Valoración económica de dicha técnica de extracción

- IV: Grado de concienciación ambiental e información socioeconómica

En el bloque I se realizan dos preguntas que permiten entablar un primer contacto con el entrevistado y conocer su opinión sobre determinados problemas que afectan a la sociedad y al medio ambiente. El bloque II, incluye cinco cuestiones complejas en las que se pregunta de forma sistemática por el conocimiento previo que manifiestan los encuestados sobre la técnica, su opinión sobre si debe estar permitida, así como la importancia que otorgan a sus beneficios, posibles riesgos y recomendaciones que se podrían llevar a cabo para minimizar su impacto. A continuación, en el bloque III se recogen las tres preguntas destinadas a la valoración económica, mediante el método de $\mathrm{VC}$, de la disposición al pago por una posible moratoria en la implementación de la técnica en la Región de Murcia. Por último, el bloque IV está destinado a conocer la información socioeconómica del encuestado y su grado de concienciación ambiental, midiendo para este último caso sus tres compromisos ecológicos (afectivo, verbal y real) (FRAJ et al, 2004).

El método de VC es una técnica utilizada en la economía ambiental que permite valorar una gran variedad de bienes, servicios y productos para los que no existe mercado a través de la simulación de un mercado hipotético mediante la realización de encuestas a los consumidores potenciales del mismo (COLINO y MARTÍNEZ-PAZ, 2010). En ellas, se les pregunta por la máxima disposición a pagar por el bien o servicio si tuvieran que adquirirlo, lo que permite valorar a éste en términos monetarios, o su mínima disposición a ser compensado por renunciar al mismo. Los fundamentos teóricos de este método son ampliamente tratados en múltiples manuales especializados, remitiendo al lector interesado a trabajos como los de MITCHELL y CARSON (1989) o AZQUETA (2002).

Un ejercicio de VC se puede estructurar en 9 fases consecutivas (RIERA, 1994): (1) determinar con precisión lo que se desea valorar en unidades monetarias, (2) definir la población objetivo, (3) concretar los elementos de la simulación del mercado, (4) decidir la modalidad de entrevista, (5) seleccionar la muestra, (6) redactar el cuestionario, (7) realizar las entrevistas, (8) analizar estadísticamente los datos y (9) presentar e interpretar los resultados. El caso que nos ocupa tiene las características recogidas en la tabla 1.

La formulación de las cuestiones relativas a la disposición a pagar (DAP) implica, en este caso, que los impactos negativos que ocasionaría la implantación de la técnica son mayores que los beneficios que se derivarían de la misma. Así, las respuestas a la pregunta sobre la disposición o no al pago ("disposición a pagar binaria", DAPB) son un indicador de la proporción de individuos que rechazan su puesta en marcha en la región. De otro lado, las respuestas a la cantidad máxima que se estaría dispuesto a aportar ("disposición a pagar total", DAPT) mediante un canon mensual en el recibo eléctrico, al estilo del que actualmente se aplica por conceptos tales como la moratoria nuclear o el fomento de las energías renovables, cuantifican dicho rechazo, de forma que el monto total obtenido se puede considerar equivalente a la valoración económica del uso de técnicas de obtención de energía con menores impactos ambientales potenciales. 
TABLA 1

Ficha técnica del estudio.

\begin{tabular}{|l|l|}
\hline \multicolumn{1}{|c|}{ Características } & \multicolumn{1}{|c|}{ Descripción } \\
\hline $\begin{array}{l}\text { Método de simulación del } \\
\text { mercado hipotético }\end{array}$ & Encuestas personales \\
\hline Método de valoración & Pregunta en formato abierto \\
\hline $\begin{array}{l}\text { Pregunta formato binario } \\
\text { de la disposición a pagar }\end{array}$ & $\begin{array}{l}\text { ¿Estaría usted dispuesto a pagar un canon mensual adicional en } \\
\text { su recibo de la electricidad a cambio de que una moratoria en la } \\
\text { implantación de la técnica de extracción de gas mediante } \\
\text { fractura hidráulica en la Región? }\end{array}$ \\
\hline $\begin{array}{l}\text { Pregunta formato abierto } \\
\text { de la disposición a pagar }\end{array}$ & $\begin{array}{l}\text { De acuerdo con sus posibilidades económicas, ¿cuánto pagaría } \\
\text { como máximo cada año por esta razón? } \text { E/año }\end{array}$ \\
\hline Población objetivo & 535.335 hogares de la Región de Murcia (INE, 2014) \\
\hline Tipo de Muestreo & $\begin{array}{l}\text { Muestreo aleatorio simple (donde cada miembro de la } \\
\text { población tiene la misma probabilidad de ser elegido) }\end{array}$ \\
\hline $\mathrm{N}^{\circ}$ encuestas piloto & 10 encuestas (Realizadas en Marzo de 2014) \\
\hline $\mathrm{N}^{\circ}$ encuestas definitivas & 369 encuestas (Realizadas en Abril de 2014) \\
\hline Error de muestreo & $\begin{array}{l}5,10 \% \text { para proporciones intermedias y 3,06\% para } \\
\text { proporciones extremas, Para un nivel de confianza del 95\% }\end{array}$ \\
\hline Material de apoyo & $\begin{array}{l}\text { Folleto informativo que recoge en qué consiste la técnica de } \\
\text { extracción, donde se está previsto desarrollar en la Región y } \\
\text { cuáles son los principales beneficios y riesgos de la misma }\end{array}$ \\
\hline
\end{tabular}

La encuesta piloto permitió testar la validez del cuestionario y corregir la formulación/redacción de algunas preguntas del mismo en su versión final.

\section{RESULTADOS Y DISCUSIÓN}

En este apartado se presentan y discuten los principales resultados, obtenidos del análisis de las respuestas a la encuesta presentada en la sección anterior, estructurados en cuatro apartados: perfil del encuestado, nivel de compromiso ecológico, estudio univariante de la disposición al pago y modelización multivariante de dicha disposición.

\subsection{Perfil del encuestado}

En primer lugar, y a modo de síntesis, se recoge el perfil del encuestado que se obtiene del análisis descriptivo de las cuestiones socioeconómicas, las preguntas sobre cuestiones generales del bloque I y las específicas sobre el tema de la fracturación hidráulica que se recogían en el bloque II.

La muestra tratada se compone de un 57\% hombres. La edad media del encuestado es de 37 años y vive en un núcleo familiar de 3 integrantes. Posee o está cursando estudios universitarios (43\%), su situación laboral mayoritaria es la de ocupado (55\%) y su renta familiar media es de $1.763 € / \mathrm{mes}$, con una renta por persona de $574 € / \mathrm{mes}$. Su domicilio habitual es de tipo urbano o urbano-periférico (83\%) y no pertenece a ningún grupo o asociación ecologista (93\%). Mayoritariamente consideran que la situación económica actual es el problema de mayor gravedad que atañe a la sociedad (4,4 sobre un máximo de 5), y que en el caso de problemas medioambientales el mayor es la mala gestión y escasez del agua (4,3 sobre un máximo de 5).

Respecto a las cuestiones específicas sobre la técnica de fracturación hidráulica, señalar que casi las dos terceras partes de la muestra $(65 \%)$ no conocían nada/muy poco sobre la técnica de extracción de gas no convencional mediante fractura hidráulica antes de acceder al material de apoyo que acompañaba a la encuesta. Tras analizar esta información un $63 \%$ opina que su uso debe estar permitido siempre y cuando la salud y el impacto medioambiental sean salvaguardados, un $20 \%$ la rechazan por completo, solo poco más del $5 \%$ se muestran 
abiertamente favorables a su implantación y el restante $12 \%$ manifiesta no tener una opinión al respecto.

En cuanto a la valoración otorgada a los beneficios potenciales del fracking que se presentan en el cuestionario, los encuestados opinan que los de mayor importancia son: permite crear empleo (señalada por el $42 \%$ de los encuestados), obtener una energía más barata (37\%), constituye una fuente de energía alternativa y más limpia que el carbón y petróleo (32\%) y disminuiría la dependencia energética del exterior (25\%). Del mismo modo, de los riesgos o impactos negativos, la cantidad de agua utilizada (señalada por el $46 \%$ de los encuestados), la calidad del agua que retorna (53\%) y la falta de transparencia e información pública del proceso de implantación (56\%), son los problemas que los encuestados consideran más graves.

De la lista de recomendaciones/medidas que se presentan para tratar de minimizar los impactos, las mejor valoradas son: evaluar previamente los riesgos antes de proceder con la actividad (señalada por el $61 \%$ de los encuestados), supervisar la afección sobre el agua, aire y la sismicidad con un plan de vigilancia que se extienda después del cese de la explotación (63\%) y garantizar un plan y una respuesta adecuada en caso de emergencia (63\%).

\subsection{Nivel de compromiso ecológico}

Dado el alto componente ambiental del tema abordado se ha medido el nivel de compromiso ecológico de los encuestados para relacionarlo seguidamente con su disposición al pago en los análisis multivariantes. Con este fin se han formulado 9 cuestiones, recogidas en la Tabla $\mathrm{n}^{\mathbf{0}} 2$, que miden las tres esferas (afectiva, verbal y real) de dicho compromiso con las actitudes proambientales (FRAJ et al., 2004). El encuestado ha señalado su nivel de acuerdo con dichas afirmaciones según una escala de Likert que va desde 1 (total desacuerdo) a 5 (total acuerdo), obteniéndose en la muestra los valores medios y la mediana recogida en la tabla 2.

TABLA 2

Afirmaciones que componen los índices de compromiso ecológico.

\begin{tabular}{|c|c|c|}
\hline A) COMPROMISO AFECTIVO. & Mediana & Media \\
\hline $\begin{array}{l}\text { - Me enfurece pensar en los daños causados al medio ambiente por } \\
\text { intereses económicos }\end{array}$ & 4 & 4,1 \\
\hline $\begin{array}{l}\text { 2. Me indigna el no apoyo gubernamental al desarrollo de energías } \\
\text { renovables }\end{array}$ & 4 & 4,0 \\
\hline 3. Creo que se exagera mucho sobre el tema del deterioro ambiental & 2 & 2,2 \\
\hline B) COMPROMISO VERBAL. & Med & Media \\
\hline $\begin{array}{l}\text { 4. Participaría en programas de voluntariado ambiental para promover el } \\
\text { uso de energías renovables }\end{array}$ & 3 & 2,8 \\
\hline $\begin{array}{l}\text { 5. Estaría dispuesto a utilizar productos menos contaminantes aunque } \\
\text { éstos cuesten más }\end{array}$ & 3 & 3,3 \\
\hline $\begin{array}{l}\text { 6. Nunca pagaría un canon ambiental para el apoyo de medidas efectivas } \\
\text { para la reducción del cambio climático }\end{array}$ & 2 & 2,6 \\
\hline MISO REAL. & Mec & $\mathrm{Me}$ \\
\hline $\begin{array}{l}\text { 7. Estoy pendiente de las propuestas ambientales del partido al que vote } \\
\text { en las últimas elecciones }\end{array}$ & 2 & 2,3 \\
\hline $\begin{array}{l}\text { 8. He asistido a una conferencia sobre un tema medioambiental en el } \\
\text { último año }\end{array}$ & 1 & 1,9 \\
\hline $\begin{array}{l}\text { 9. Soy consumidor de productos ecológicos y/o respetuosos con el } \\
\text { medioambiente }\end{array}$ & 3 & 2,1 \\
\hline
\end{tabular}

La información de dichas cuestiones se puede sintetizar en tres índices (uno por cada tipo de compromiso) con solo tomar la media de sus tres ítems, una vez corregida la escala de las cuestiones 3 y 6 , de forma que todas ellas, y por tanto los índices, sean del tipo a más acuerdo 
mayor compromiso. La descriptiva de los tres índices para el conjunto de la muestra es la recogida en la tabla 3.

TABLA 3

Índices de compromiso ecológico.

\begin{tabular}{|l|c|c|c|c|c|c|}
\hline \multicolumn{1}{|c|}{ Índices } & $\mathrm{N}$ & Mínimo & Máximo & Media & Desv. Típ. & Intervalos de confianza (95\%) \\
\hline C. Afectivo & 369 & 1 & 5 & 3,96 & 0,895 & $2,3-5,0$ \\
\hline C. Verbal & 369 & 1 & 5 & 3,19 & 0,938 & $1,7-4,7$ \\
\hline C. Real & 369 & 1 & 5 & 2,31 & 1,050 & $1,0-4,3$ \\
\hline
\end{tabular}

El mayor valor corresponde al compromiso ecológico afectivo $(3,96)$ seguido del verbal $(3,19) \mathrm{y}$, por último, el real $(2,31)$. Estos datos muestran que los encuestados tienen un alto grado de concienciación ambiental (compromiso afectivo) y que la mayoría de ellos estarían dispuestos a realizar algunas acciones respetuosas con el medio ambiente en su vida cotidiana (compromiso verbal), pero en realidad son menos los encuestados que llevan a cabo de forma integral las acciones descritas en el cuestionario (compromiso real).

Cabe suponer que aquellos encuestados que presentan un mayor compromiso ecológico afectivo, presentarán un mayor compromiso verbal y real, y a su vez que estos dos últimos también tendrán un comportamiento paralelo, de forma que los tres índices de compromiso estén relacionados. Para comprobarlo se ha realizado un análisis de correlación entre los índices (tabla 4) que demuestra que existe una correlación positiva y estadísticamente significativa entre los mismos, confirmando la hipótesis de partida, lo cual es a su vez prueba de validez del proceso de encuesta.

TABLA 4

Correlación y significación entre los índices de compromiso ecológico.

\begin{tabular}{|c|c|c|c|}
\cline { 2 - 4 } \multicolumn{1}{c|}{} & Afectivo - Verbal & Afectivo - Real & Verbal - Real \\
\hline Coef. de Pearson & 0,583 & 0,422 & 0,497 \\
\hline Significación & 0,000 & 0,000 & 0,000 \\
\hline
\end{tabular}

Los valores medios de los índices calculados en este trabajo son muy similares a los obtenidos con una metodología similar, pero con distintas cuestiones en su formulación, en otros estudios realizados en la Región de Murcia como los tres casos recogidos en PERNI et al. (2012) y el trabajo de MARTÍNEZ-PAZ et al. (2011).

\subsection{Estudio univariante de la disposición al pago}

En este apartado se presenta en primer lugar el análisis de la proporción de encuestados que están o no dispuestos a pagar a cambio de que se prohíba la extracción de gas mediante fractura hidráulica en la Región de Murcia. Una primera lectura de las respuestas indica que aproximadamente una cuarta parte (96 encuestados) estaría dispuesta a realizar esta contribución (DAP+), mientras que el resto (273 encuestados) no lo estaría (DAP=0). Ahora bien, estos porcentajes deben ser analizados con más profundidad ya que dentro de aquellos que se muestran no dispuestos, hay que distinguir los llamados "no reales" de los conocidos como "no protesta". Los primeros son aquellos que realmente no valoran el bien o servicio ofrecido y por tanto no estarían dispuestos a contribuir a su financiación. Los segundos son aquellos cuya respuesta "cero" no está indicando que no valoren el bien, sino que recoge actitudes negativas frente al escenario de valoración y consecuentemente dan un valor de cero cuando en realidad su valor verdadero es positivo. Estos últimos casos, cuya respuesta no refleja su verdadera valoración, no se consideran como válidos para obtener el valor del bien/servicio en cuestión (RIERA, 1994). Con el fin de distinguir entre ambos tipos de "no" se pidió a los encuestados que daban dicha respuesta que indicaran el motivo/s, obteniendo los resultados presentados en la tabla 5. 
TABLA 5

Motivos de la disposición al pago igual a cero.

\begin{tabular}{|l|c|c|}
\hline \multicolumn{1}{|c|}{ Motivos de la DAP = } & $\mathrm{n}$ & $(\%)$ \\
\hline El control de la técnica es competencia directa de las AAPP & 120 & 43,96 \\
\hline No dispongo de renta suficiente para pagar más, aunque me importa el tema & 61 & 22,34 \\
\hline Ya dono dinero para otras iniciativas de protección del medio y la salud & 10 & 3,66 \\
\hline No considero apropiado prohibir su implementación & 29 & 10,62 \\
\hline No considero apropiado el vehículo de pago & 66 & 24,18 \\
\hline A la hora de realizar una aportación, considero otros temas más prioritarios & 47 & 17,22 \\
\hline Otros & 320 & 11,72 \\
\hline
\end{tabular}

Considerando como respuestas protesta las habituales en la literatura sobre el tema (RIERA, 1994), que son las de aquellos individuos que argumentan que el control de la técnica es competencia de la Administración Pública y/o no consideran adecuado el vehículo de pago, surge el mercado hipotético representado en la figura 2, en el que las respuestas válidas para el ejercicio de valoración pasan a ser 204, una vez retirados los 165 individuos que ofrecen un no protesta. La alta tasa de respuestas protesta viene explicada por la misma naturaleza del bien que estamos valorando, con posibles consecuencias directas sobre la salud, etc, por lo que muchos encuestados ven en el control de la técnica una obligación de la Administración Pública.

La primera conclusión es que los encuestados sí dispuestos a pagar y los que no lo están se reparten en igual proporción en el mercado. Así cabe afirmar que, y según este método de valoración, la mitad de la población de la Región de Murcia rechaza la implementación de la técnica de fracturación hidráulica ya que estarían dispuestos a contribuir económicamente para que no se autorizara.

FIGURA 2

Composición del mercado hipotético.

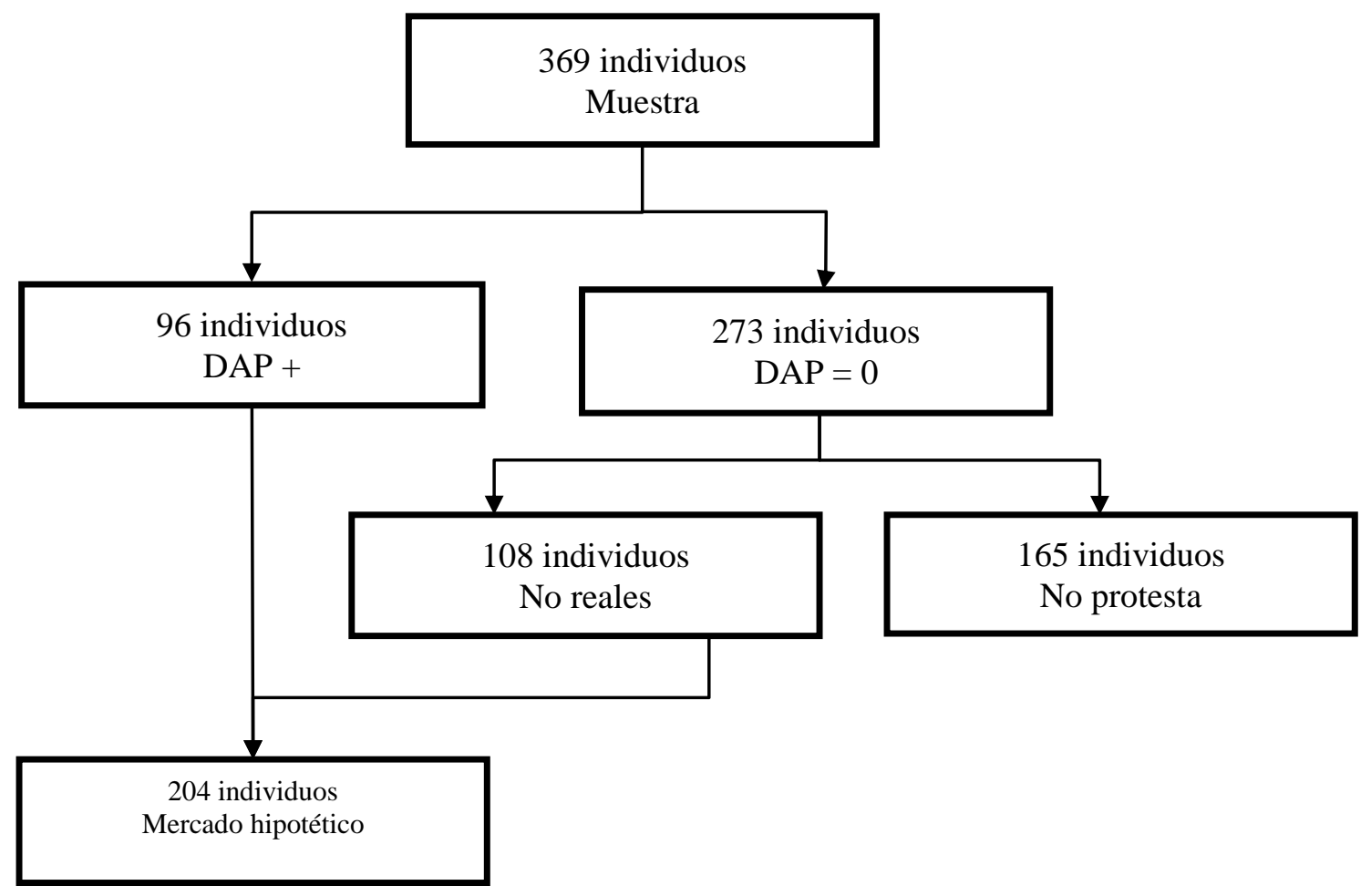


Pasando ahora a cuantificar el monto de dicha disposición, dado por la estadística descriptiva de la respuesta abierta (DAPT) se obtienen los resultados mostrados en la tabla 6, con la distribución de frecuencias recogida en la figura 3.

\section{TABLA 6}

Estadísticos principales de la DAP (€/año).

\begin{tabular}{|c|c|c|c|c|c|}
\hline $\mathrm{n}$ & Media & Mediana & Mínimo & Máximo & Desv. Típica \\
\hline 204 & 15,48 & 0 & 0 & 200 & 27,963 \\
\hline
\end{tabular}

El valor medio de dicha DAPT es de 15,48 €/año, cifra que representaría un incremento en torno al 1,6 \% para una facturación de la electricidad media de unos $80 € /$ mes, porcentaje cercano, al por ejemplo correspondiente a la parte de los impuestos especiales del recibo eléctrico destinada a la moratoria nuclear, que se puede cifrar en el 1,2\% (CNE; 2013). Por su parte, si el valor medio de la DAPT obtenido se extrapola al total de la población objetivo (los 535.335 hogares que componen la Región de Murcia) el monto total ascendería a 8.286.986 $€ /$ año.

FIGURA 3

Distribución de frecuencias de la DAP (€/año).

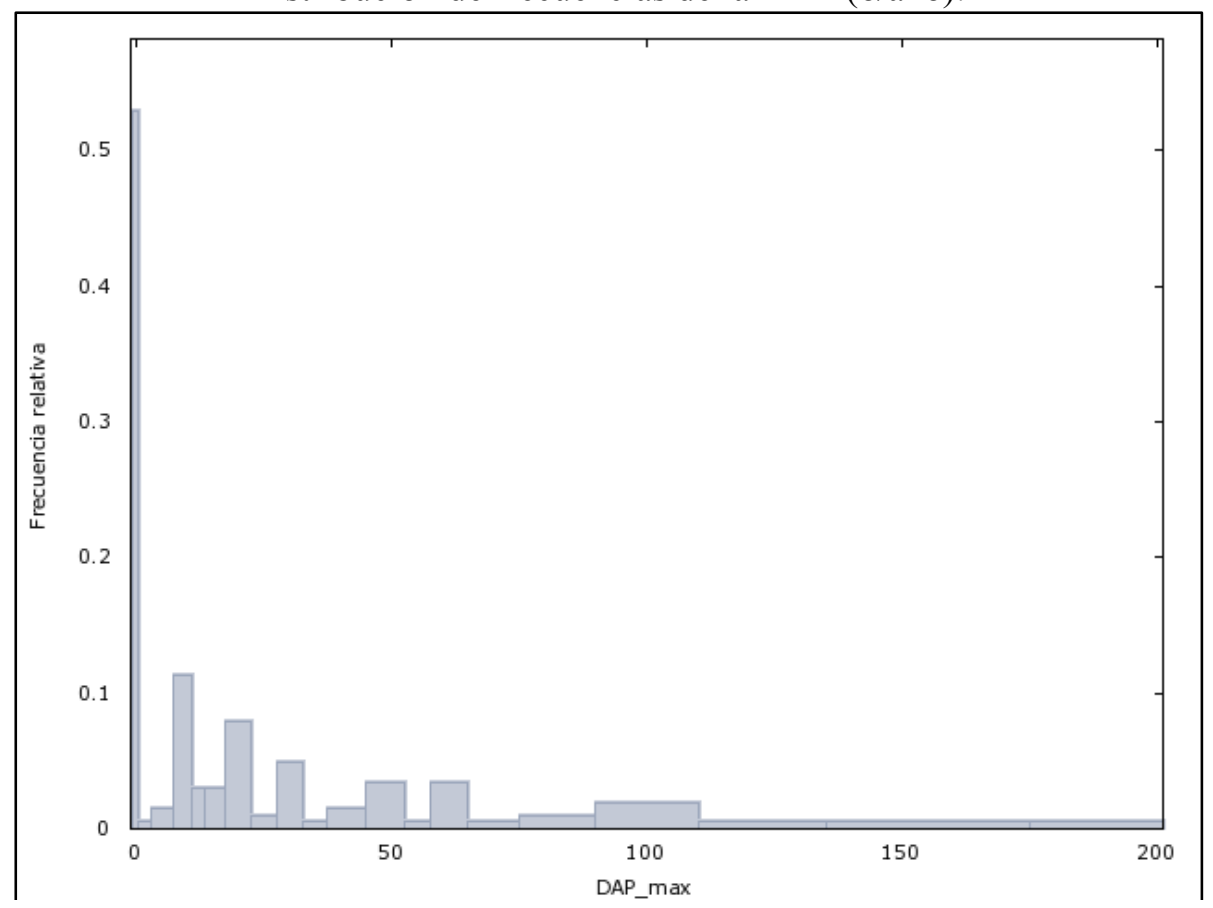

\subsection{Modelización multivariante de la DAP}

Con la finalidad de estudiar las motivaciones y características que hacen que los ciudadanos de la Región de Murcia estén dispuestos o no a pagar (DAPB) para que no se autorice la técnica de la fracturación hidráulica, así como las que influyen en la cuantía de dicho pago (DAPT), se han estimado dos modelos multivariantes donde se relacionan cada tipo de disposición con posibles factores explicativos.

Para el primer caso, dada la naturaleza binaria de la variable de DAPB se ha estimado un modelo logit binario multivariante (GREENE, 1997). Para el caso de la DAPT se recurre a la estimación de una regresión tobit censurada en cero por la izquierda ya que la variable dependiente tiene estas características (AMEMIYA, 1985), como se constata en el histograma de la Figura $\mathrm{n}^{\mathbf{0}}$ 3. Las variables independientes que se han considerado a priori para ambos modelos han sido las características socioeconómicas del encuestado (sexo, edad, renta,...), los 
tres índices de compromiso ecológico, la proximidad o no de su lugar de residencia a las zonas con mayor potencial/disponibilidad de gas no convencional (Altiplano y Noroeste de la Región de Murcia) y sus niveles de conocimiento previo sobre la técnica de extracción.

Para la disposición a pagar binaria (DAPB) las variables explicativas que han resultado significativas son (tabla 7):

- ZONA: Variable binaria que toma un valor de 1 si el individuo vive en una localidad de la Región de Murcia próxima a las zonas que podrían verse afectadas por la técnica de extracción

- CON: Conocimiento sobre la técnica de extracción. Toma un valor de 1 si el individuo conoce algo o bastante sobre la técnica y 0 si no ha escuchado prácticamente nada acerca de la misma.

- ICV: Índice de compromiso ecológico verbal. Es una variable continua que toma valores del 1 al 5 como ya se indicó en epígrafes anteriores.

TABLA 7

Estimación logit para la DAPB.

\begin{tabular}{|c|c|c|c|c|c|}
\hline Variable & Coeficiente & Desv. Típica & $\mathrm{z}$ & Valor $\mathrm{p}$ & Pendiente \\
\hline Constante & $-2,940$ & 0,648 & $-4,535$ & 0,000 & - \\
\hline ZONA & 0,636 & 0,387 & 1,643 & 0,100 & 0,158 \\
\hline CON & 0,721 & 0,311 & 2,318 & 0,020 & 0,178 \\
\hline ICV & 0,707 & 0,184 & 3,847 & 0,000 & 0,176 \\
\hline \multicolumn{7}{|c|}{ Número de casos correctamente predichos $=139(68,1 \%)$} \\
\hline
\end{tabular}

El modelo resulta significativo en su conjunto y tiene una capacidad de predicción elevada dado su elevado porcentaje de clasificación correcta. Las variables independientes no presentan problemas de multicolinealidad, dado el bajo valor de su FIV (GREENE, 1997), y presentan el signo esperado. De la interpretación del modelo puede deducirse que:

1) Los individuos cuya localidad de residencia está en las zonas del Altiplano y Noroeste de la Región de Murcia tienen una probabilidad de estar dispuestos a pagar un 15,8\% mayor que el resto.

2) Los individuos que conocen mejor la técnica de extracción tienen una probabilidad de estar dispuestos a pagar un $17,8 \%$ mayor que si no la conocen.

3) Un aumento de una unidad en el índice de compromiso ecológico verbal (ICV) supone un incremento del $17,6 \%$ de la probabilidad de que los individuos del mercado hipotético estén dispuestos a pagar.

Pasando a la disposición a pagar total (DAPT), los resultados de la estimación recogidos en la tabla 8 muestran que la cuantía total es explicada por las siguientes variables:

- CON: Conocimiento sobre la técnica de extracción.

- ICA: Índice de compromiso ecológico afectivo. Variable continua que toma valores del 1 al 5 como ya se indicó en epígrafes anteriores.

- SEX: Variable referida al sexo. Toma un valor de 0 si el individuo es un hombre y 1 si es una mujer.

- RFM: Variable que hace referencia a la renta familiar mensual del individuo encuestado. 
TABLA 8

Estimación Tóbit de la DAPT.

\begin{tabular}{|c|c|c|c|c|}
\hline Variable & Coeficiente & Desv. Típica & $\mathrm{z}$ & Valor $\mathrm{p}$ \\
\hline constante & $-110,130$ & 22,700 & $-4,852$ & 0,000 \\
\hline CON & 22,676 & 7,734 & 2,932 & 0,003 \\
\hline ICA & 18,672 & 5,031 & 3,711 & 0,000 \\
\hline SEX & 13,391 & 7,668 & 1,746 & 0,081 \\
\hline RFM & 0,007 & 0,004 & 2,043 & 0,041 \\
\hline \multicolumn{5}{|c|}{ Contraste de razón de verosimilitudes: Chi-cuadrado(2) $=10,480(0,005)$} \\
\hline
\end{tabular}

El modelo es significativo dado el contraste de razón de verosimilitudes, no presenta problemas de colinealidad y los signos de las variables no contradicen los esperados a priori. Dado el carácter lineal de este modelo, del mismo se puede concluir que: 1) las personas que conocen la técnica de extracción pagarían al año 22,68€/año más que aquellas personas que no la conocen; 2) el aumento de una unidad en el ICA de un individuo, supone un incremento de $18,67 € /$ año en su DAPT; 3) las mujeres presentan una disposición a pagar anual 13,39€/año mayor que la presentada por los hombres; y 4) un aumento de $1.000 € /$ mes en la renta familiar mensual del encuestado incrementaría $7 € /$ año su DAPT. Cabe señalar la no significatividad de la variable ZONA en este modelo, con lo que la proximidad geográfica a las áreas de explotación explicaría el hecho de estar dispuesto a pagar, pero no la cuantía de dicho pago.

\section{CONCLUSIONES}

A continuación se presentan a modo de resumen y discusión las consideraciones más importantes obtenidas en este trabajo sobre el conocimiento, percepción y aceptabilidad que los ciudadanos de la Región de Murcia tienen sobre la extracción de gas no convencional mediante fractura hidráulica.

El conocimiento de la técnica entre los murcianos es en términos absolutos bajo, ya que poco más de un tercio de los mismos conocía siquiera su existencia y menos de una décima parte afirma conocer bastante sobre el tema. Ahora bien, este porcentaje es bastante similar al obtenido en otras latitudes. Por ejemplo, el estudio de POPKIN (2012) realizado en Estados Unidos concluye que solo el $40 \%$ de los ciudadanos que viven en estados sin explotación de fracking afirman conocer esta técnica, porcentaje que se eleva al $84 \%$ en los que si tiene zonas de extracción ya consolidas.

Tras informarse con el material de apoyo que acompaña la encuesta, hay un estado de opinión mayoritariamente favorable a permitir el uso de la técnica siempre y cuando la salud y el medioambiente sean salvaguardados. Sin embargo, mientras una quinta parte rechaza su implementación en la región, apenas uno de cada veinte murcianos la apoya en cualquier caso. Estas últimas opiniones se confirman en el análisis cuantitativo de la disposición a pagar por la prohibición de la implementación de la técnica de fracturación hidráulica en la Región de Murcia, ya que la mitad de su población estaría dispuesta a contribuir económicamente para que no se autorizara. La cuantía media resultante de dicho pago, de algo más de $15 €$ /año por hogar en el recibo eléctrico, representa un sobrecoste muy cercano al que se paga actualmente como impuestos especiales en apoyo a otras políticas energéticas.

Los índices de compromiso ecológico obtenidos muestran, como es habitual en estudios similares, que los compromisos afectivo y verbal son elevados, que las personas tiene una alta sensibilidad (afecto) por la protección del medio ambiente, se declaran dispuestos a realizar acciones con este fin (verbalización) pero su nivel de actuación (realización) es menor. Así, resulta lógico que en la modelización de la DAP, que no es sino la verbalización de una disposición, tanto el índice de compromiso verbal (en el caso del monto total) como el afectivo (para la disposición binaria) han resultado ser dos variables significativas, pero no lo ha sido el compromiso real en ninguno de los casos. 
Del análisis de los motivos para estar o no dispuestos a pagar, se puede inferir la necesidad de que la Administración Pública tome un papel activo en la investigación y evaluación de los riesgos ambientales y sobre la salud humana de la implantación de la técnica. En el caso de la Región de Murcia, zona de alto riesgo sísmico y donde el agua es un recurso escaso, ambos impactos habrían de ser tenidos especialmente en consideración.

De la modelización de ambas DAP cabe destacar dos aspectos. En primer lugar como el hecho de residir en una localidad de la Región de Murcia próxima a las zonas que podrían verse afectadas por la técnica de extracción aumenta el rechazo a su implantación en torno al $16 \%$, un efecto vecindad ya constatado en estudios similares en otras zonas del mundo (BOUDET, 2011; BENNET y LOOMIS, 2015). De otro lado las mujeres murcianas son más propensas a oponerse al fracking, al resultar la variable sexo significativa en el modelo explicativo monto de la DAPT, al igual que ocurre con las ciudadanas norteamericanas como señalan BOUDET et al. (2014).

Por lo tanto, aunque el crecimiento económico en las últimas décadas ha estado asociado a un notable aumento del consumo energético, y a pesar de que el crecimiento de la producción norteamericana de gas parece demostrar que la explotación del gas no convencional puede ser económicamente viable (CÁMARA y PENDAS, 2013), es prioritario tener presentes las posibles repercusiones que esta técnica podría ocasionar en el medio ambiente, en el bienestar de las personas y conocer la percepción social que se tiene de la misma en cada uno de los lugares donde se quiera desarrollar. Campañas de información previas, dirigidas a la sociedad en general y basadas en información técnica rigurosa contrastada a nivel local serían muy necesarias para fomentar la participación pública en la formulación de planes de explotación del gas no convencional. Las campañas que se viene realizando, tanto por organizaciones civiles de oposición a la fracturación hidráulica como por compañías energéticas concesionarias, son en algunos casos sesgadas y/o basadas en experiencias en otras áreas geográficas no necesariamente extrapolables respecto a los impactos ambientales y socioeconómicos de la explotación de este tipo de energía no renovable.

\section{BIBLIOGRAFÍA}

ACIEP. (2012): Evaluación preliminar de los recursos prospectivos de hidrocarburos convencionales y no convencionales en España. Informe de la Asociación española de compañías de investigación, exploración y producción de hidrocarburos. $17 \mathrm{pp}$.

AMEMIYA, T. (1985): Advanced Econometrics. Basil Blackwell, Oxford, 536 pp.

AZQUETA, D. (2002): Introducción a la Economía Ambiental. McGraw-Hill, Madrid, 456 pp.

BENNETT, A. y LOOMIS, J. (2015): Society \& Natural Resources. "Are Housing Prices Pulled Down or Pushed Up by Fracked Oil and Gas Wells?", (in press). doi: 10.1080/08941920.2015.1024810.

BOE. (2013): Ley 21/2013, de 9 de diciembre, de evaluación ambiental. Boletín Oficial del Estado, $n^{\circ}$ 296, referencia: BOE-A-2013-12913.

BOE. (2015): España. Ley 8/2015, de 21 de mayo, por la que se modifica la Ley 34/1998, de 7 de octubre Boletín Oficial del Estado, $\mathrm{n}^{\circ}$ 122, referencia: BOE-A-2015-5633.

BOUDET, H. (2011): Environmental Politics. "From NIMBY to NIABY: regional mobilization against liquefied natural gas in the United States", vol. 20, p. 786-806.

BOUDET, H., CLARKE, C., BUGDEN D., MAIBACH E., ROSER-RENOUF, C. у LESIDERWOTIZ, A. (2014): Energy Policy. “"Fracking» controversy and communication: using national survey data to understand public perceptions of hydraulic fracturing", vol. 65, p. 57-67.

CÁMARA, A. y PENDAS, F. (2013): Gas no Convencional en España, una Oportunidad de Futuro. Informe del Consejo Superior de Colegios de Ingenieros de Minas de España. Madrid. 149 pp. 
CHS. (2013): Borrador del Plan de Cuenca de la Demarcación del Segura. Confederación Hidrográfica del Segura. Ministerio de Agricultura, Alimentación y Medio Ambiente. Murcia.

CNE. (2013): Boletín mensual de indicadores eléctricos y económicos. Comisión Nacional de Energía. Dirección de Regulación y Competencia. Ministerio de Economía y Competitividad de España. 103 pp.

COLBORN, T., Schultz, K., HERRICK, L. y KWIATKOWSKI, C. (2014): Human and Ecological Risk Assessment. "An exploratory study of air quality near natural gas operations", 20(1), p. 86-105.

COLINO, J. y MARTÍNEZ-PAZ, J. M. (2010): El valor económico de los espacios naturales. "Análisis ecológico, económico y jurídico de la Red de Espacios Naturales en la Región de Murcia". En Esteve, M.A., Martínez-Paz, J.M., Soro B. (Ed.). Editum, Murcia, pp. 211- 240.

COOLEY, H. y DONNELLY, K. (2012): Hydraulic Fracturing and Water Resources: Separating the Frack from the Fiction. Pacific Institute. Oakland, 35 pp.

DOUE. (2014): Recomendación de la comisión de 22 de enero de 2014 relativa a unos principios mínimos para la exploración y producción de hidrocarburos (como el gas de esquisto) utilizando la fracturación hidráulica de alto volumen. (2014/70/UE) Diario Oficial de la Union Europea n ${ }^{\circ} 39$ L 39/72 DOUE 8.2.2014. 7 pp.

EC. (2013): Analysis and presentation of the results of the public consultation "Unconventional fossil fuels (e.g. shale gas) in Europe". European Commission DG Environment Final report. $142 \mathrm{pp}$.

EIA. (2013): Technically Recoverable Shale Oil and Shale Gas Resources: An assessment of 137 shale formations in 41 countries outside the United States. U.S. Department of Energy. United Stated of America Energy Information Administration. 730 pp.

EPA (2015): Assessment of the Potential Impacts of Hydraulic Fracturing for Oil and Gas on Drinking Water Resources. EPA/600/R-15/047a, June 2015 http://www.epa.gov/hfstudy

FRAJ, E. MARTÍNEZ, E. y GRANDE, I. (2004): Revista de Economía y Empresa. "Un estudio exploratorio sobre las variables psicográficas que influyen en el comportamiento del consumidor ecológico", vol. 50, n 21, 61-87.

GARCÍA-PORTERO, J. (2012): El gas natural no convencional. El gas natural como energía puente entre el presente energético y el deseable futuro sostenible. XI Congreso Nacional del Medio Ambiente (CONAMA 2012). Madrid, 32pp.

GERLACH, J. (2013): Chicago Policy Review. "Natural Gas: A Bubble of Expectation?" Disponible en http://chicagopolicyreview.org/2013/03/19/natural-gas-a-bubble-ofexpectation/

GREENE, W. H. (1997): Econometric Analysis. Prentice-Hall, New Jersey, 827 pp.

INE. (2014). Datos de Población. Disponible en: www.ine.es

KASSOTIS, C., TILliTT, D., DAVIS, J., HORMANN, A. AND NAGEL, S. (2014). Endocrinology. "Estrogen and Androgen Receptor Activities of Hydraulic Fracturing Chemicals and Surface and Ground Water in a Drilling-Dense Region", vol. 155(3), pp.897907.

MARTíNEZ, J. (2011): Papeles de Geografía. "Sismos del 11 de mayo de 2011 en Lorca", vol. 53-54, pp. 199-213.

MARTÍNEZ-PAZ, J. M., ALMANSA-SÁEZ, C. y PERNI-LLORENTE, A. (2011): Estudios de economía aplicada. "Energía eléctrica procedente de fuentes renovables: Percepción social y disposición al pago", vol. 29(2)1, pp. 539 - 560.

MATESANZ, J. (2013): Repercusiones territoriales de la fractura hidráulica o fracking en Cantabria, Burgos y Palencia. Documento de trabajo E- prints Complutense. Universidad Complutense de Madrid. 141pp. Disponible en http://eprints.ucm.es/23795/

MITCHELL, R. y CARSON, R. T. (1989): Using Survey to Value Public Goods: the Contingent Valuation Method. John Hopkins University Press, Baltimore, 488 pp. 
MINETUR (2015) Mapa de posición de sondeos, permisos y concesiones. Ministerio de Industria, Energía y Turismo de España. Disponible en: http://www.minetur.gob.es/energia/petroleo/Exploracion/Mapa/Paginas/mapSondeos.aspx

PEINADO, M. (2014): El ecologista .La burbuja del Fracking. Vol ${ }^{\circ}$ 82, otoño 2014.

PERNI A., MARTÍNEZ, R., FERRERES, E., FRUCTUOSO, E., NAVALON, E. y ORTUÑO, A. (2012): Contexto social de la valoración económica de parques regionales en la región de Murcia. En Esteve Selma, M. A.; Martínez Paz, J. M. y Soro Mateo, B. (Eds). "Análisis ecológico, económico y jurídico de la red de espacios naturales en la región de Murcia". Editum. Murcia, pp. 241-262.

POPKIN, J. (2012): Willingness to pay for electricity sourced from natural gas extracted using hydraulic fracturing: location and preference heterogeneity. Thesis of Master of Science. University of Delaware, Delaware, $95 \mathrm{pp}$.

POPKIN, J.., DUKE, J., BORCHERS, A. y ILVENTO, T. (2013): Energy Policy. "Social costs from proximity to hydraulic fracturing in New York State", vol. 62, p. 62-69.

RAHM, B. y RIHA, S. (2012): Environmental Science \& Policy. "Toward strategic management of shale gas development: regional, collective impacts on water resources", vol. 17, p. 12-23.

RATNER, M., PARFOMAK, P., LUTHER, L. y FERGUSSON, I. (2015): U.S. Natural Gas Exports: New Opportunities, Uncertain Outcomes. Congressional Research Service Report R42074, 32 pp.

RIERA, P. (1994): Manual de Valoración Contingente. Instituto de Estudios Fiscales. Madrid, $188 \mathrm{pp}$.

ROUSU, M., RAMSARAN, D. y FURLANO, D. (2015): International Advances in Economic Research. "Guidelines for Conducting Economic Impact Studies on Fracking", vol. 21, no 2, p. 213-225.

SÁNDEZ, J. (2014): La fracturación hidráulica en la Unión Europea: estado de la cuestión. Instituto Español de Estudios Estratégicos (IEEE). Documento marco de IEE no 18/24. Ministerio de Defensa, $30 \mathrm{pp}$.

VENGOSH, A., JACKSON, R., WARNER, N., DARRAH, T. y KONDASH, A. (2014): Environmental Science \& Technology. "A Critical Review of the Risks to Water Resources from Unconventional Shale Gas Development and Hydraulic Fracturing in the United States", vol. 48(15), p. 8334-8348.

WESTAWAY, R., y YOUNGER, P. L. (2014): Quarterly Journal of Engineering Geology and Hydrogeology. "Quantification of potential macroseismic effects of the induced seismicity that might result from hydraulic fracturing for shale gas exploitation in the UK", vol. 47, p. 333-350. 